Mappemonde

Revue trimestrielle sur l'image géographique et les

formes du territoire

$131 \mid 2021$

Varia

\title{
Visualisation de l'évolution du trafic aérien passager et marchandises entre 2009 et 2019
}

\section{Vincent Vivanloc}

\section{OpenEdition}

Journals

Édition électronique

URL : https://journals.openedition.org/mappemonde/6335

DOI : $10.4000 /$ mappemonde.6335

ISSN : 1769-7298

Éditeur

UMR ESPACE

\section{Référence électronique}

Vincent Vivanloc, « Visualisation de l'évolution du trafic aérien passager et marchandises entre 2009 et 2019 », Mappemonde [En ligne], 131 | 2021, mis en ligne le 08 juillet 2021, consulté le 14 juillet 2021. URL : http://journals.openedition.org/mappemonde/6335; DOI : https://doi.org/10.4000/ mappemonde.6335

Ce document a été généré automatiquement le 14 juillet 2021.

\section{cc) (†)}

La revue Mappemonde est mise à disposition selon les termes de la Licence Creative Commons Attribution - Pas d'Utilisation Commerciale - Partage dans les Mêmes Conditions 4.0 International. 


\title{
Visualisation de l'évolution du trafic aérien passager et marchandises entre 2009 et 2019
}

\author{
Vincent Vivanloc
}

\section{Contexte et introduction}

1 Cette application, créée dans le cadre du Mapathon $2020^{1}$, présente l'évolution du trafic aérien passager et marchandise entre 2009 et 2019 (figures 1 et 2). Les trafics sont figurés par un graphe spatial non orienté sur deux cartes de France, une pour les passagers et l'autre pour les marchandises. Les sommets correspondent à l'agrégation des trafics des différents aéroports d'une même ville et les arcs représentent les flux entre ces dernières. Cette application est visible en ligne $^{2}$, depuis un navigateur internet, depuis un ordinateur, mais aussi une tablette ou un smartphone. 
Figure 1. Vue statique de l'application pour 2009

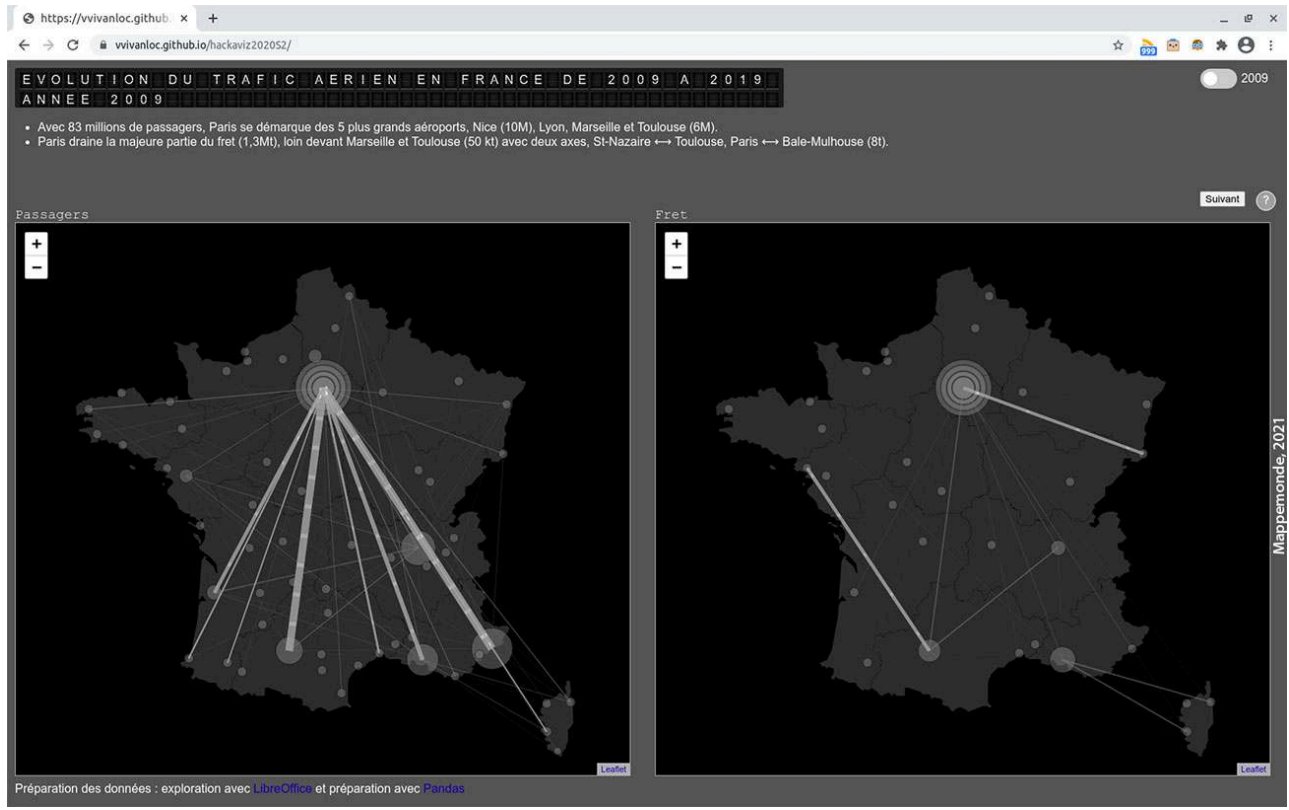

Figure 2. Vue statique de l'application pour 2019

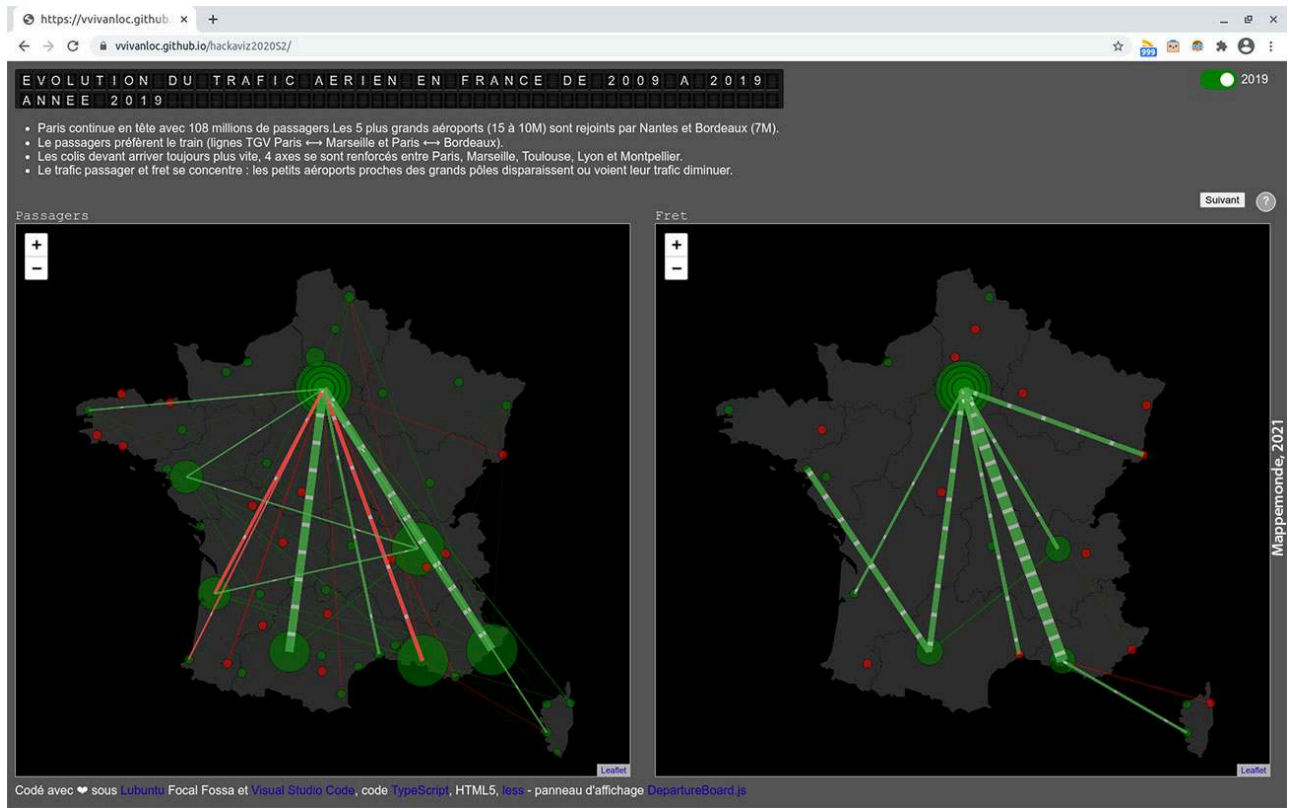

\section{Description}

2 L'interaction sur les deux cartes s'effectue à l'aide de modalités bien établies par Google Maps depuis 2005 (Miller, 2006) : cliquer-glisser pour se déplacer et zoom par molette souris ou pincement sur écran tactile. La navigation se déroule en parallèle sur les deux cartes, passagers et marchandises pour une année donnée : l'action de centrer la vue sur une région délimitée sur la carte des passagers est répercutée sur la vue des marchandises. Cela permet d'explorer les différences entre les flux de passagers et de fret à différentes échelles. 
3 Un bouton permet de basculer l'affichage entre les deux années afin de mettre en évidence l'évolution entre 2009 et 2019. Explorer ces données m'a permis de déconstruire mes préjugés sur le trafic aérien; le trafic aérien passager n'est pas en croissance constante et uniforme dans le pays et j'ai trouvé des axes de circulation de fret que je ne soupçonnais pas. Ces premières observations sont résumées, pour l'année sélectionnée, dans un descriptif affiché en encart sur l'application. Libre à l'utilisateur d'interpréter cette évolution du trafic selon ses propres observations et connaissances au cours de ses explorations.

\section{Réalisation}

4 La contrainte de temps imposée par le concours m'a conduit à me restreindre à présenter les données selon un point de vue bien délimité. Le jeu de données proposait des valeurs mensualisées sur toutes les années de 2009 et 2020. Mon propos étant de comparer le trafic aérien sur une durée de 10 ans, j'ai choisi d'agréger les données passagers et fret pour les deux seules années 2009 et 2019. J'ai aussi volontairement omis l'année 2020, les données étant incomplètes sur cette année, et la situation sanitaire et économique liée au COVID étant exceptionnelle.

Des premiers essais m'ont montré que je pouvais afficher toutes données sur la carte, le jeu de données du Mapathon ayant été écrêté. Toutefois, représenter les passagers et les flux simultanément rendait la carte illisible : je me suis résolu à représenter ces informations sur deux cartes différentes (Tufte, 1990). Ces deux cartes utilisent un fond de carte épuré et monochrome, version simplifiée issue de la base Khortis ${ }^{3}$; ce fond ne contient que les contours des régions françaises sur un fond gris. Le trafic étudié étant restreint à la France, les pays frontaliers et les océans ne sont pas représentés.

6 La taille en pixels des rayons des sommets et la largeur des arcs sont un compromis entre la lisibilité de la carte et l'ergonomie de l'application. Le rayon des disques associés aux villes et la largeur des segments figurant les échanges entre deux villes sont proportionnels aux trafics respectifs. D'une part, pour assurer la lisibilité de la carte, le trafic des aéroports parisiens étant de 10 à 23 fois supérieur respectivement en nombre de passagers et en fret, le motif choisi pour Paris est différent (Bertin, 1973). D'autre part, une taille minimale a été fixée pour que les petits aéroports puissent être pointés avec la souris 4 .

7 Une animation ajoutée sur les arcs illustre le trafic entre deux villes: des particules figurant des avions circulent aléatoirement entre les deux localisations. À la place de ces points, des symboles sous forme d'avion ont été envisagés. Sur le site FlightRadar24 $4^{5}$, cette iconographie est justifiée, le symbole correspondant à un avion positionné dans l'espace aérien à un instant donné. Pour mon application, le trafic étant quantifié par un cumul annuel, une telle iconographie n'aurait pas amélioré la compréhension de la visualisation. Une seconde animation sous la forme d'un affichage dynamique, rappelant le tableau général ${ }^{6}$ des départs des aéroports des années 1980, a été toutefois conservée : étant limitée au titre de l'application, elle ne nuisait pas à la lisibilité des cartes.

8 Les couleurs choisies sont le gris, le vert et le rouge, couleurs utilisées en navigation aérienne ${ }^{7}$. Le gris figure les données du passé, de 2009 ; ensuite en 2019, le vert signifie une augmentation du trafic et le rouge, une diminution. Suite au retour du jury du 
concours, une légende expliquant ce choix de couleurs et un mode d'emploi ont été ajoutés à la version initiale de l'application.

9 Sur le plan technique, cette application est un site web statique: après le téléchargement des données sur la machine de l'utilisateur lors de la première visite, aucune communication entre le client et un serveur. Afin de réduire la taille des données géographiques, ces dernières sont extraites du jeu de données du Mapathon à l'aide de la bibliothèque Python Pandas. Cette bibliothèque permet d'importer un jeu de données tabulaire et de le traiter à l'aide d'opérations d'algèbre relationnelle (filtrage, projection et jointure). Le rendu de la carte interactive est assuré par le pendant open source de Google Maps, Leaflet ${ }^{8}$. L'animation des particules et le déplacement en parallèle sur les deux cartes de France ont été réalisés à l'aide de deux greffons. À l'instar des autres solutions open source utilisées lors de la réalisation de cette visualisation, l'ensemble des sources de ma contribution est disponible sur GitHub ${ }^{9}$ afin que le lecteur puisse les télécharger et expérimenter à son tour.

\section{Conclusion et perspectives}

La réalisation de cette application m'a permis de découvrir l'évolution du trafic aérien entre 2009 et 2019. J'espère que le lecteur, en explorant les données, pourra à son tour trouver des comparaisons intéressantes. J'ai découvert de nouvelles techniques et j'ai pu progresser dans l'art de la géovisualisation.

11 Une représentation tridimensionnelle a été envisagée, mais par manque de temps, elle n'a pas été réalisée. Remplacer un disque de rayon proportionnel au trafic, par une hauteur de cylindre permettrait de changer de point de vue sur la différence entre Paris et les différentes villes. Par ailleurs, une extension de l'application à l'année 2020 permettrait d'observer l'impact de la crise sanitaire sur le trafic aérien.

\section{BIBLIOGRAPHIE}

BERTIN J. (1973). Sémiologie graphique. Les diagrammes, les réseaux, les cartes. Paris, La Haye : Mouton/ Gauthier-Villars, p. 42-43.

MILLER C.C. (2006). "A Beast in the Field: The Google Maps Mashup as GIS/2”. Cartographica: The International Journal for Geographic Information and Geovisualization, vol. 41, nº 3, p. 187-199.

TUFTE E. (1990). Envisioning Information. Graphics Press, p. 67.

\section{NOTES}

1. AFIGEO 2020, Colloque Tous (im)mobiles, tous cartographes, concours de (géo)datavisualisation de données, Mapathon 2020 (cartomob.sciencesconf.org).

2. Vincent Vivanloc, application Mapathon 2020 https://vvivanloc.github.io/hackaviz2020S2/ 
3. Fonds de cartes Khorthis : www.sciencespo.fr/cartographie/khartis/docs/les-fonds-de-cartedisponibles/

4. Steven Hoober, 2013, Common Misconceptions About Touch. www.uxmatters.com/mt/ archives/2013/03/common-misconceptions-about-touch.php

5. FlightRadar24, Live Air Traffic, https://www.flightradar24.com

6. Afficheur à palette : fr.wikipedia.org/wiki/Afficheur_\%C3\%A0_palettes

7. Balisage : fr.wikipedia.org/wiki/Balisage\#LLe_syst\%C3\%A8me_lat\%C3\%A9ral

8. Vladimir Agafonkin et al., 2020, Leaflet, an open-source JavaScript library for mobile-friendly interactive maps, https://leafletjs.com

9. Vincent Vivanloc, sources Mapathon 2020 : github.com/vvivanloc/hackaviz2020S2 